\title{
EDUQUITO: FERRAMENTAS ACESSÍVEIS NO PROCESSO DE ENSINO/APRENDIZAGEM DE UM ALUNO COM PARALISIA CEREBRAL
}

\author{
Tatiana Lima dos Santos da Cunha - UFRGS - cunha.tatiana@uol.com.br \\ Lucila Costi Santarosa - UFRGS - lucilamcs@yahoo.com.br \\ José Valdeni de Lima - UFRGS - valdeni@inf.ufrgs.br
}

\section{RESUMO}

O presente trabalho se propõe a identificar as possíveis contribuições da utilização de ferramentas acessíveis de ambientes virtuais de aprendizagem, aqui o Eduquito, no processo de ensino aprendizagem de um aluno com paralisia cerebral. O trabalho surgiu de uma inquietação sobre as novas tecnologias beneficiando pessoas com deficiência. Estudo de caráter qualitativo, na forma de estudo de caso de um único sujeito, apoiada nos pressupostos propostos por Vygotsky. O sujeito foi orientado a utilizar o ambiente, durante a sessão fonoaudiológica. Foram avaliadas as evoluções dentro do ambiente, nas ferramentas, na leitura e escrita e na linguagem oral. Não foram identificados outros estudos para comparar os benefícios do uso do Eduquito no âmbito dos aspectos fonoaudiológicos. O sujeito obteve evolução em todas as ferramentas do ambiente, conseguindo maior autonomia e independência dentro do ambiente utilizado. Obteve melhora significativa na leitura e na escrita, ampliou seu vocabulário, porém suas alterações de fala permaneceram iguais. Os resultados obtidos puderam contribuir para o trabalho fonoaudiológico junto às crianças com paralisia cerebral, trazendo novas perspectivas de atendimento e também contendo informações importantes, despertando o interesse para o início de novas e mais completas pesquisas que visem ampliar os conhecimentos sobre o tema proposto.

\section{Palavras-chaves}

Ambientes virtuais de aprendizagem; informática na educação; fonoaudiologia; paralisia cerebral.

\begin{abstract}
This study aims to identify possible contributions of the use of virtual learning environments, Eduquito here, in the teaching learning of a student with cerebral palsy. The work grew out of a concern about new technologies benefiting people with disabilities. Qualitative study in the form of a case study of a single subject, based on the assumptions proposed by Vygotsky. The student was asked to use the environment Eduquito, in speech therapy session. We evaluated the changes in the environment, tools, reading and writing and oral language. No other studies were identified to compare the benefits of using Eduquito aspects within the speech therapy. The student got all the tools in the evolution of the environment, achieving greater autonomy and independence within the environment used. Significant improvement in reading and writing, expanded vocabulary, but their speech disorders remained the same. The results could contribute to the speech therapy with children with cerebral palsy, bringing new perspectives of care and also containing important information, arousing interest for the beginning of new and more comprehensive research aimed at increasing knowledge about the theme.
\end{abstract}

\section{Keywords}

Virtual learning environments, computers in education; speech; cerebral palsy. 


\section{INTRODUÇÃO}

Este estudo surgiu da necessidade de se utilizar um Ambiente Virtual de Aprendizagem (AVA), como um recurso no processo de ensino/aprendizagem de um sujeito com Paralisia Cerebral (PC). Sabe-se que a informática, de um modo geral, auxilia na educação destes alunos, porém não existem estudos que comprovem que a comunicação das pessoas com deficiência seja influenciada pelo uso de AVA inclusivos e de suas ferramentas. Os recursos convencionais disponíveis hoje em dia, conseguem envolver um indivíduo, porém os recursos digitais podem se transformar em um ambiente mais atrativo, mais interativo, e como consequência, pode se tornar mais eficiente na utilização de pessoas com deficiência.

A comunicação é a forma que se tem de interagir, trocar informações, conhecimentos, e compartilhar sensações, pensamentos e sentimentos. Estudos, como o de Tijiboy et al. (2002) apontam para o benefício que o uso dos recursos da informática traz as pessoas com deficiência. De acordo com Siqueira e Santana (2010), a efetivação da inclusão de pessoa com deficiência requer clareza sobre a própria condição da pessoa, de suas habilidades e fragilidades. Neste estudo buscamos as eficiências do aluno para favorecer seu aprendizado.

Este estudo esta baseado em uma concepção sócio-histórica, a partir da teoria Vygotskiana. Na qual a organização e a estruturação das funções psíquicas superiores se formam no processo do desenvolvimento social do sujeito a partir das interações e colaborações com o meio social (Vygotsky, 2007). Atualmente, é possível relacionar o enfoque teórico do desenvolvimento social do ser humano com propostas de aprendizagem coletiva. Alguns AVA valorizam a abordagem colaborativa, pautada nas teorias do desenvolvimento sócio-cognitivo e afetivo das pessoas. As ferramentas de comunicação síncronas e assíncronas, que suportam as interações privilegiam a colaboração entre os participantes, possibilitando trocas afetivas, discussão, compartilhamento. Com o uso abundante das tecnologias, a acessibilidade aumentou de tal forma, que hoje se observa a utilização do computador em diversas classes sociais, bem como a utilização por diversos tipos de pessoas, dentre eles as pessoas com deficiências.

O computador pode ser visto como um grande instrumento na melhora da qualidade de vida de sujeitos com PC, pois através das diversas adaptações possíveis, pode tornar-se importante agente facilitador da aprendizagem, do desenvolvimento, e instrumento imprescindível na possibilidade de comunicação, assumindo assim o papel de fundamental promotor da inclusão social desses indivíduos. A fim de oferecer uma forma estruturada e organizada de recursos de informação e comunicação para mediação do processo de ensino e aprendizagem, o uso das Tecnologias de Informação e Comunicação (TIC) feito por meio dos AVA, é fundamental. Em um levantamento abrangente e crítico da literatura, realizado por Chantry e Dunford (2010), sobre o impacto do uso de computadores por crianças com deficiências, os resultados apoiaram o uso das tecnologias com crianças dos mais variados graus de dificuldades, em especial, as tecnologias favoreceram as áreas da comunicação, educação e atividades lúdicas. O uso crescente das tecnologias digitais amplifica uma profunda mutação na relação com o saber. De acordo com Levy (1999) quando prolonga-se determinadas capacidades cognitivas humanas, como a memória, imaginação ou percepção, é possível que as tecnologias intelectuais com suporte digital redefinam o seu alcance, seu significado e sua natureza em determinados casos. A comunicação é uma necessidade que está presente na vida do ser humano. A troca de informações entre as pessoas está diretamente relacionada com a qualidade da interação. Novas tecnologias foram criadas a partir das necessidades que foram surgindo ao longo dos anos. 
A apropriação das TIC em virtude da inclusão sociodigital, bem como do favorecimento da comunicação de indivíduos deficientes, traz a tona estudos que comprovam os benefícios da utilização das tecnologias digitais como recurso no processo de construção de conhecimentos. Alguns estudos, como os de Santarosa e Basso (2008) e Santarosa et al. (2007) desenvolvidos pelo Núcleo de Informática na Educação Especial (NIEE) mostraram resultados favoráveis neste sentido. É com base nestes estudos a justificativa desta pesquisa, que tem como objetivo, estudar o processo de interação, apropriação e comunicação oral e escrita de um sujeito com PC.

\section{MATERIAL E MÉTODO}

\subsection{Amostra}

O presente estudo foi caracterizado como pesquisa qualitativa de estudo de caso, com um único sujeito. O sujeito selecionado para este estudo estava em atendimento fonoaudiológico individual com a pesquisadora/terapeuta, paciente do gênero masculino, com 17 anos, aluno da CP - Turma de Progressão do terceiro ciclo da Escola Municipal Professor Gilberto Jorge Gonçalves da Silva, alfabetizado e com diagnóstico de PC, sem causa definida. O sujeito escolhido já possuía conhecimento prévio de informática básica, com boa fluência digital, porém com certa dificuldade e não possuía nenhum conhecimento sobre o ambiente a ser utilizado. O sujeito apresentava dificuldades de leitura e escrita, bem como alterações de linguagem e fala. Apresentava ótimo vínculo com terapeuta, e boa convivência com sua família, e era extremamente bem relacionado com amigos e pessoas estranhas. Sujeito estava em atendimento fonoaudiológico há sete anos.

\subsection{Método}

O sujeito incluído na pesquisa foi estimulado a utilizar o AVA Eduquito, durante a terapia fonoaudiológica, tendo a pesquisadora/terapeuta como mediadora. Foram realizadas 15 sessões fonoaudiológicas, no período de setembro a novembro de 2008, totalizando três meses. Algumas das ferramentas disponíveis no ambiente Eduquito não foram utilizadas, apenas as que o sujeito teve condições de utilizar durante este período. O correio e o chat foram realizados com a própria pesquisadora/terapeuta. Os demais recursos foram utilizados pelo próprio sujeito, apenas tendo a pesquisadora/terapeuta como mediadora. A intervenção da mediadora se fez presente em todas as etapas, de forma a intervir sempre que necessário, para atender às necessidades do sujeito em sua evolução em todo o processo.

\subsection{Considerações Éticas}

A participação do sujeito no estudo foi realizada de acordo com a resolução 196/96 do Conselho Nacional de Saúde (aspectos éticos de pesquisa em seres humanos), através da assinatura do Termo de Consentimento Livre Esclarecido, pelo responsável pela mesma. Foi mantido o sigilo e a confidenciabilidade dos dados pesquisados sobre o sujeito, bem como as informações vinculadas à identificação da mesma. Foi dada autorização por escrito pelos pais do sujeito para a utilização de vídeos, fotos, dados pessoais e mensagens para a realização e exposição deste trabalho.

\subsection{Recurso utilizado}

O AVA escolhido para este estudo foi o Eduquito, o qual teve sua elaboração fundamentada na abordagem sóciointeracionista proposta por Vygotsky, o que permitiu investigar como a interação social promove o desenvolvimento de Funções 
Psicológicas Superiores no desenvolvimento da vida de um indivíduo (Santarosa e Basso, 2007).

Alguns estudos posteriores apresentados por Santarosa e Basso (2009) e Santarosa et al (2010), apresentam o Eduquito com a proposta de ser um espaço virtual de inclusão digital/social onde todos possam exercer sua cidadania em termos de convivência e desenvolvimento de projetos colaborativos, em áreas de interesse comum. Além disto, o Eduquito permite o desenvolvimento de atividades que motivam o processo de crescimento individual e coletivo. No estudo aqui realizado o AVA Eduquito foi usado como propósito de utilizar ferramentas que permitam esta troca de conhecimento através da mediação e da interação social.

O ambiente Eduquito, dentro das concepções teóricas anteriormente referidas, foi projetado sobre um modelo baseado na web, visando à facilidade de acesso e uso, a interface amigável, a interatividade e a independência da localização geográfica. A única necessidade para acesso ao sistema é que o usuário esteja conectado à Internet e que possua em seu computador um browser (navegador). O acesso via browser permite, além da facilidade de utilização, maior portabilidade ao ambiente, uma vez que os usuários poderão acessá-lo de forma independente do Sistema Operacional de sua preferência (Windows, Linux, Mac). (Santarosa et al, 2007)

O Eduquito dispõem em sua estrutura interna, três áreas. Na área superior, apresenta-se a barra de acessibilidade. Na área esquerda encontra-se o menu de ferramentas, necessárias para o desenvolvimento do projeto. O espaço central é destinado à apresentação do conteúdo correspondente a ferramenta habilitada pelo usuário.

No menu de ferramentas, existem duas opções de visualização: visão do participante e visão do coordenador (ou mediador). Na visão do participante, algumas ferramentas não são apresentadas, uma vez que esses usuários têm seu acesso com algumas restrições que somente cabem ao coordenador do projeto decidir o que ele usará. As ferramentas do ambiente estão descritas abaixo, na ordem que estão apresentadas no ambiente.

Projeto - ferramenta utilizada pelo coordenador para que os demais usuários visualizem a sua proposta ou projeto; Agenda de Atividades - ferramenta que tem a finalidade de explicitar as atividades que serão desenvolvidas pelos usuários no decorrer do projeto. Apenas o coordenador tem acesso à edição da agenda; Midiateca funciona como um repositório de materiais produzidos ou anexados, os quais podem ser compartilhados com os participantes do projeto. Os materiais armazenados na Midiateca podem ter diferentes formatos - áudio, vídeo, imagem, texto ou outros tipos de arquivos; Oficina Multimídia - é uma das últimas ferramentas desenvolvidas, permite a criação, individual ou coletivamente, de documentos multimídia com texto, imagem, som e vídeo. Informações Urgentes - espaço para deixar um material considerado importante para as atividades do projeto; Quadro de Avisos - ferramenta para deixar postados, por qualquer usuário, avisos que se tornam visíveis para todos; Fórum ferramenta para discussão sobre temas de interesse. Podem ser abertos por qualquer participante do projeto; Chat - sala de bate-papo, ficando também o registro no ambiente. Qualquer participante pode marcar uma sessão de bate-papo; Correio ferramenta de correio eletrônico tradicional (e-mail) interno, ficando o registro no ambiente; Grupo de Trabalho - ferramenta também de cunho organizacional destinada a permitir a formação de grupos de usuários para o desenvolvimento de atividades dentro do ambiente. Qualquer participante poderá criar um grupo de trabalho e convidar participantes; Quem Sou - espaço onde os usuários incluem seus dados pessoais e outras informações para que os demais participantes o conheçam. É compartilhado com todos os participantes do ambiente; Meu Diário de Bordo funciona como um diário onde os participantes podem registrar suas alegrias e/ou 
frustrações relacionadas a todo o contexto; Espaço de Produção - local onde os usuários podem incluir trabalhos ou materiais pessoais, os quais podem ou não ser compartilhado com os demais participantes. Nessa ferramenta, também podem ser armazenados os trabalhos desenvolvidos pelos eventuais grupos formados dentro de um projeto;

Existem duas novas ferramentas do AVA Eduquito que já estão em fase de testes, o Bloguito e o Quadro-Branco. Segundo Santarosa et al. (2011), o Bloguito possui um recurso externo ao ambiente de aprendizagem, possibilitando que o aluno possa interagir fora do ambiente. Um dos propósitos do Quadro-Branco é ser uma ferramenta síncrona para a autoria coletiva que impulsiona o desenvolvimento de habilidades para a atuação em rede, para a produção da inteligência coletiva para e pela diversidade humana. O objetivo destas ferramentas é instrumentalizar sujeitos com deficiências para o uso de tecnologias de comunicação e de informação, desenvolvendo habilidades para atuação em rede, para a produção da inteligência coletiva e pela diversidade humana. (Santarosa et al. 2012)

\subsection{Instrumento de observação}

Foi elaborado um protocolo de observação, baseado nas ferramentas do ambiente Eduquito. Este protocolo não continha todas as ferramentas do ambiente, apenas as que foram trabalhadas. O protocolo foi preenchido em cada sessão fonoaudiológica, com o intuito de se observar o desenvolvimento e a evolução do sujeito. Após o uso de cada ferramenta, foram descritos os aspectos fonoaudiológicos e os relacionados ao processo de ensino/aprendizagem. As ferramentas observadas durante o estudo foram: Agenda, Midiateca, Chat, correio, Quem sou e Espaço de produção.

\section{RESULTADOS E DISCUSSÃO}

\subsection{Conhecimentos básicos}

O sujeito foi avaliado quanto aos seus conhecimentos básicos de informática. Durante todas as sessões observou-se que o sujeito conseguiu uma melhora na autonomia das atividades, conquistando uma maior independência em todo o processo envolvido durante a sessão.

O sujeito conseguiu uma melhora na velocidade de digitação e melhora na capacidade de identificar alguns ícones utilizados em atividades gerais de informática. Antes deste estudo, o sujeito nunca havia entrado no ambiente e não possuía nenhum conhecimento prévio sobre este ambiente utilizado. Sua autonomia foi sendo criada durante cada sessão realizada. De acordo com Vygotsky (2007) é através das relações interpessoais concretas com os outros homens que o indivíduo interioriza as formas culturalmente estabelecidas. Foi por meio desta interação social com os outros, no caso com a pesquisadora/terapeuta - mediadora, que o sujeito obteve informações sobre o funcionamento do ambiente, conseguindo após esta interação aprimorar os novos conceitos, internalizando o significado das palavras, ícones e símbolos.

\subsection{Ferramentas do ambiente}

Após entrar no ambiente, o sujeito conquistou uma autonomia muito grande em todo o ambiente Eduquito e não apenas em uma ferramenta específica. A melhora do processo de ensino/aprendizagem foi notória, beneficiando o sujeito em diversos aspectos da aprendizagem. Observou-se que a tecnologia utilizada, por ser diferente, atraiu a atenção do sujeito para as atividades. A introdução do computador na educação, segundo Santarosa (2004), tem provocado uma revolução na concepção de ensino e de aprendizagem. A quantidade de programas educacionais e as diferentes possibilidades 
de uso do computador mostram que esta tecnologia pode ser bastante útil no processo de ensino aprendizagem, e à medida que este uso se propaga, consolida-se como uma ferramenta de complementação, de aperfeiçoamento e de possível mudança na qualidade do ensino.

Inicialmente o sujeito não identificava os ícones e não apresentava uma boa interação com o ambiente, após algumas sessões, foi se familiarizando e conseguiu manter uma boa independência dentro do ambiente, conseguindo seguir as orientações que continham na AGENDA. O AVA Eduquito tem a acessibilidade fundamentada como um dos seus pilares de desenvolvimento (Santarosa e Basso, 2008). Esta ferramenta demonstra-se como um recurso com enorme potencial para explorar o desenvolvimento sujeitos envolvidos em processos de aprendizagem, uma vez que possibilita o trabalho em grupo, estimula a criatividade e capacidade de abstração. Todas estas características são importantíssimas para o desenvolvimento de qualquer indivíduo, mas apresentam um papel ainda mais fundamental para os sujeitos com deficiência.

Após já estar mais familiarizado com o ambiente, o sujeito conseguiu, através de outros programas de informática, criar imagens para enviar a MIDIATECA. O desenho foi realizado no programa Paintbrush.

Na primeira sessão que se utilizou o CHAT, o sujeito e a pesquisadora/terapeuta interagiram juntos, utilizando apenas 1 usuário no chat. Nesta sessão ele foi instruído a utilizar as ferramentas e a como interagir durante uma conversação pelo chat. A figura 2 mostra uma parte da primeira utilização do chat.

Figura 2 - Primeiro contato com o Chat

Início: $11: 17: 48$

Fim: 11:28:15

(11:17:48) LAURO Entra na sala...

(11:18:05) LAURO fala para Todos: OI

(11:18:19) LAURO fala para Todos: INTER

(11:19:46) LAURO fala para Todos: AVIÃO

(11:22:05) LAURO fala para Todos: अّINTE2006

(11:23:12) LAURO fala para Todos:

(11:23:57) LAURO fala para Todos:

(11:25:46) LAURO fala para Todos:

(11:26:13) LAURO fala para Todos: PTUDO BEM?

(11:26:39) LAURO fala para Todos:

(11:28:04) LAURO fala para Todos: ${ }^{\circ}$ TUDO TATI

(11:28:15) LAURO Sai da sala...

A figura mostra que o sujeito ainda não conseguiu manter uma interação adequada, seus comentários não eram pertinentes e estavam fora do contexto. Com o passar do tempo e com um maior conhecimento da ferramenta, o sujeito conseguiu melhorar seu texto e sua formatação. Na figura 3, o sujeito já demonstra maior autonomia, conseguindo interagir sozinho na frente do computador. Neste chat já existiam 2 usuários conectados, o sujeito e a pesquisadora/terapeuta.

O sujeito conseguiu com a utilização do chat, melhorar sua escrita e sua leitura. Conseguiu maior autonomia para trabalhar sozinho em um computador, necessitando da pesquisadora/terapeuta, apenas para as questões mais específicas. 
Figura 3 - Chat com 2 usuários

\begin{tabular}{|l|}
\hline Início: $08: 21: 33$ / Fim: 08:50:16 \\
\hline$(08: 21: 33)$ LAURO Entra na sala... \\
$(08: 22: 04)$ TATIANA Entra na sala... \\
$(08: 22: 35)$ TATIANA fala para Todos: OI LAURO \\
$(08: 27: 15)$ LAURO fala para Todos: OI TATIANA \\
$(08: 29: 03)$ TATIANA fala para Todos: VIU O JOGO DO INTER? \\
$(08: 30: 41)$ LAURO fala para Todos: SIN \\
$(08: 36: 21)$ LAURO fala para Todos: LAURO CANPEAO JAPAO 2006. \\
$(08: 36: 29)$ TATIANA fala para Todos: QUANTOS GOLS O INTER FEZ \\
$(08: 38: 09)$ LAURO fala para Todos: UM GOL 1 \\
$(08: 40: 12)$ TATIANA fala para Todos: MUITO BEM! \\
$(08: 41: 45)$ LAURO fala para Todos: QUEM FES O GOL \\
$(08: 42: 42)$ TATIANA fala para Todos: FOI O ALEX \\
$(08: 44: 38)$ LAURO fala para Todos: A CERTOU \\
$(08: 46: 38)$ TATIANA fala para Todos: VAMOS DESLIGAR? \\
$(08: 47: 59)$ LAURO fala para Todos: SIM TATI \\
$(08: 48: 17)$ TATIANA fala para Todos: \\
$(08: 50: 14)$ LAURO fala para Todos: TCHAU \\
$(08: 50: 15)$ LAURO Sai da sala... \\
$(08: 50: 16)$ TATIANA Sai da sala...
\end{tabular}

De acordo com Vygotsky, as crianças crescem imersas em um ambiente de constante interação com os adultos, e oferecem ao mundo, respostas dominadas pelos processos naturais. É através da constante mediação dos adultos, que processos mentais mais complexos começam a tomar forma. Inicialmente, estes processos funcionam durante a interação das crianças com os outros, principalmente os adultos, os quais servem de mediadores no contato da criança com o mundo (Santarosa et al. 2007). No decorrer do crescimento, estes processos acabam por ser executados internamente pelas crianças. Esta constatação corrobora com nossos achados, pois mostra que os processos, através da interação com o mediador, passam a ser executados internamente após serem aprendidos. Da mesma forma, um estudo realizado por Rocha e Deliberato (2012), mostrou que o ambiente inclusivo favoreceu a interação do aluno com PC com outros interlocutores competentes em diversas habilidades.

A utilização do CORREIO foi explorada com sucesso. O sujeito já possuía um conhecimento prévio da utilização de correio eletrônico, e o fácil acesso ao correio do ambiente favoreceu para que o sujeito não tivesse maiores dificuldades em interagir pelo correio. Inicialmente o correio serviu apenas para testagem, e após, conseguiu-se manter um diálogo bem mais sucedido.

Na ferramenta QUEM SOU, o sujeito conseguiu descrever seu perfil, e até incluir uma filmagem, onde ele aparece mergulhando na piscina. Inicialmente o sujeito necessitou de auxílio para saber o que significavam os ícones e onde ele deveria escrever. A cada sessão foi preenchida uma parte do perfil, e no último preenchimento ele já não precisou auxílio técnico e nem pedagógico. A cada duas sessões, uma parte era preenchida. Com o decorrer do tempo observou-se a evolução da escrita e consequentemente da leitura. $\mathrm{Na}$ ferramenta ESPAÇO DE PRODUÇÃO, o sujeito conseguiu interagir bem, nas últimas sessões, onde conseguiu disponibilizar duas imagens que fez em outro programa. 
Em um estudo realizado por Araújo e Benassi (2005) onde 14 sujeitos com deficiência foram avaliados, os autores referem sobre os benefícios que a informática traz aos conhecimentos escolares. Estes resultadas corroboram com os resultados encontrados em nosso estudo. A partir dos achados, os autores referiram que seus resultados mostraram que a informática vem proporcionando aos alunos, de um modo geral, uma melhor aquisição dos conteúdos desenvolvidos em sala de aula observados numa assimilação e construção mais efetiva destes e que uma interpretação dada para o melhor desempenho no computador fundamenta-se no fato de que a situação virtual possui uma característica de monitoramento, que permite a essas crianças ter um feedback imediato quanto às estratégias escolhidas; uma forma de aprendizagem mais científica.

Ferrada (2009) realizou um estudo com o objetivo de verificar de que forma ocorre a adaptação e apropriação da Tecnologia Assistiva em sujeitos com deficiência, em ambientes virtuais/digitais de aprendizagem, com vistas à inclusão sócio-digital. O trabalho, assim como o nosso, se baseou nas concepções sócio-histórica de Vygotsky. Neste estudo de caráter qualitativo, foram incluídos três sujeitos com deficiência. A autora concluiu que houve um processo bem sucedido de adaptação à tecnologia, possibilitando melhorias em termos de tempo e precisão de movimentos. Ressalta-se neste estudo a contribuição que o ambiente Eduquito trouxe para esta pesquisa, pela sua capacidade de unir em uma mesma plataforma o acesso às diferentes ferramentas usadas para a realização de interações através dos AVAs.

\subsection{Aspectos fonoaudiológicos}

O sujeito apresentou durante estes 15 encontros, evoluções tanto na área da leitura, como na escrita. A linguagem oral foi beneficiada, indiretamente, fazendo com o sujeito aumentasse seu vocabulário e utilizasse o Eduquito, como assunto em seus diálogos com outras pessoas.

Um estudo realizado por Ruiz (2002) avaliou um sujeito com PC, vem ao encontro com nossos achados, pois demonstrou que o aluno avaliado apresentou melhora tanto no aumento do vocabulário, quanto no desenvolvimento da expressividade. Os autores relatam que "Ao contar a história, notou-se um esforço para organizar o pensamento, contribuindo para o desenvolvimento do raciocínio. Essa atividade também cumpriu seu objetivo de estimular a fala fluente, ampliando seu vocabulário e a comunicação oral, além do desenvolvimento da expressividade e da desinibição. Além disso, a atividade pode refletir na boa convivência com os demais alunos, pessoas ou parentes, através da conversa, expressão dos desejos, necessidades, opiniões, em diversas situações do seu cotidiano”.

A leitura e a escrita foram trabalhadas em todas as sessões constantemente. O sujeito necessitava ler e escrever para poder interagir dentro do ambiente, sozinho ou tendo a pesquisadora/terapeuta como mediadora. Acredita-se que esta necessidade de utilizar estas duas funções foi um fator determinante para ele obter esta melhora.

Tijiboy et al. (2002), realizou um estudo que vem ao encontro dos nossos achados, os autores avaliaram dois sujeitos com PC em relação à apropriação das tecnologias de informação. Tal estudo mostrou que ambos sujeitos obtiveram melhora na linguagem escrita. Os autores referiram que na relação entre pensamento e linguagem, uma das premissas é que a necessidade de comunicação (primeira função da linguagem - função social) parece haver impulsionado, inicialmente na filogênese, o desenvolvimento da linguagem. Falando especificamente sobre a linguagem escrita, foi possível observar que esta premissa se aplica também no novo contexto, no ambiente computacional telemático. A necessidade ou o desejo de comunicar ideias no ciberespaço que, envolve 
ponderadamente a forma escrita, parece haver impulsionado o desenvolvimento da linguagem escrita em ambos os casos em estudo.

\section{CONCLUSÕES}

O ambiente computacional oportunizou ao sujeito envolvido no estudo, novos espaços de aprendizagem, oferecendo diferentes ferramentas e recursos de comunicação que o desafiou e o motivou a ler e escrever, em construção conjunta com outra pessoa, e até mesmo individualmente, tornando-o ativo no processo de aprendizagem e comunicação e abrindo novos caminhos para a formação de vias colaterais, como propõe Vygotsky, e construção do conhecimento. Verificou-se que no caso de indivíduos com PC a limitação motora não interferiu na criatividade e o mediador foi fundamental para dar o suporte na Zona de Desenvolvimento Próximal, para que ele pudesse desenvolver todo seu potencial criativo.

A autoestima, sentimento de satisfação com relação a si próprio e a seu desempenho na interação com o ambiente, é um elemento importante no desenvolvimento de qualquer pessoa, porém ela toma uma importância ainda maior quando se trata de seres humanos considerados deficientes pela sociedade como um todo. O sujeito desenvolveu uma capacidade de atingir seus objetivos dentro do ambiente, conseguindo sucesso nas últimas sessões, o que tornou este momento, extremamente satisfatório para o sujeito reforçando sua autoestima.

Os resultados obtidos neste estudo mostraram que o ambiente Eduquito e suas ferramentas acessíveis estimularam e ativaram as funções mentais superiores, na medida em que o sujeito interagia com o ambiente e apropriava-se dos recursos, conseguindo assim, desenvolver processos psicológicos superiores, habilidades motoras e aspectos sócio-afetivos. Espera-se que este trabalho possa despertar o interesse para novas e mais aprofundadas pesquisas, envolvendo AVA e crianças portadoras de PC.

\section{REFERÊNCIAS BIBLIOGRÁFICAS}

ARAÚJO S.L.S, BENASSI K.P. 2005. O Uso dos Recursos da Informática como Instrumento Mediador no Processo de Ensino-Aprendizagem de Pessoas com Necessidades Educativas Especiais: Um relato de Experiência. Revista Brasileira de Informática na Educação, v. 13, n. 2, p. 65-70, 2005.

CHANTRY, J.; DUNFORD, C. (2010) How do computer assistive technologies enhance participation in childhood occupations for children with multiple and complex disabilities? A review of the current literature. The British Journal of Occupational Therapy, v.73, n. 8, p. 351-365, 2010.

FERRADA, R.B.H. 2009. Inclusão Digital de sujeitos com Deficiência Física através do uso da Tecnologia Assistiva. 2009. Dissertação de Mestrado apresentada a Universidade Federal do Rio Grande do Sul. Porto Alegre, 2009.

LEVY, P. 1999. Cibercultura. São Paulo: Editora 34.

ROCHA, A. N. D.C.; DELIBERATO, D. 2012. Tecnologia Assistiva para a criança com Paralisia Cerebral na Escola: Identificação das necessidades. Revista Brasileira de Educação Especial, v.18, n.1, p. 71-92, 2012.

RUIZ, C.A. 2002. A informática como ferramenta de auxílio na inclusão do portador de paralisia cerebral: um estudo de caso. Iniciação científica: Cesumar. V. 4, n. 1, p. 37-48, 2002.

SANTAROSA, L.C.; BASSO, L.O. 2008. Oficina de Produção: uma ferramenta de escrita coletiva de documentos multimídia acessível a PNEs. Revista Novas Tecnologias na Educação, v. 6, n. 1, p. 01-10, 2008. 
SANTAROSA, L.M.C.; BASSO, L.O. 2009. Eduquito: Virtual Environment for Digital Inclusion of People with Special Educational Needs. Journal of Universal Computer Science, v. 15, p. 1496-1507, 2009.

SANTAROSA, L.C.; BASSO, L.O.; PASSERINO, L.; DIAS, C. 2007. Acessibilidade em Ambientes de Aprendizagem por Projetos: construção de espaços virtuais para inclusão digital e social de PNEEs. Revista Novas Tecnologias na Educação, v. 5, n. 1, p. 1-11, 2007

SANTAROSA, L.M.C.; CONFORTO, D.; BASSO, L. O. 2010. Eduquito: Ergonomia Cognitiva para a Diversidade Humana. Revista Educação, Formação \& Tecnologias. P. 4-13: 2010.

SANTAROSA, L.M.C.; CONFORTO, D.; LEITHARDT, V.; RIES, L.H. 2011. BLOGUITO: acessibilidade e autoria para a diversidade humana na perspectiva da web 2.0. In: ICEM-SIIE2011, 2011, AVEIRO. Anais do ICEM-SIIE2011, 2011. v. 1. p. 113. 2011. v. 1. p. 177-184.

SANTAROSA, L.M.C.; CONFORTO, D.; MACHADO, R.P. 2012. Quadro-Branco: uma Ferramenta Síncrona, Colaborativa e Acessível In: Nuevas Ideas em Informática Educativa, Anais do TISE. 2012

SANTAROSA L.M.C, HEIDRICH R. 2004. Análise do processo de inclusão de alunos com paralisia cerebral utilizando as tecnologias da informação e comunicação. In: SBIE - simpósio de Informática na Educação, 2004, Manaus, 2004.

SIQUEIRA, I. M.; SANTANA, C. S. 2010. Propostas de acessibilidade para a inclusão de pessoas com deficiências no ensino superior. Revista Brasileira de Educação Especial, v.16, n.1, p. 127-136, 2010.

TIJIBOY, A.V.; SANTAROSA, L.M.C.; TAROUCO, L.A. 2002. Apropriação das Tecnologias de Informação e Comunicação por pessoas com Paralisia Cerebral. Revista de Informática Teórica e aplicada, v. 5, n. 2, p. 131-152, 2002.

VYGOTSKY, L.S. 2007. A Formação Social da Mente: o desenvolvimento dos processos psicológicos superiores. $7^{\mathrm{a}}$ edição. São Paulo: Martins Fontes. 